\title{
Study of Hydrogen Bonding in Small Water Clusters with Density Functional Theory Calculations
}

\author{
Johanna Wendlandt \\ Office of Science, Science Undergraduate Laboratory Internship (SULI) \\ University of Wisconsin \\ Stanford Synchrotron Radiation Laboratory \\ Stanford Linear Accelerator Center \\ Menlo Park, CA
}

August 19, 2005

Prepared in partial fulfillment of the requirements of the Office of Science,

Department of Energy's SULI program under the mentorship of

Professor Anders Nilsson at the Stanford Synchrotron Radiation Laboratory

Participant:

Research Advisor: 


\begin{abstract}
The unique characteristics of hydrogen bonding have left our understanding of liquid water far from complete in terms of its structure and properties. In order to better describe the hydrogen bond in water, we seek to understand the electronic states which show sensitivity to hydrogen bonding. We investigate the structure of unoccupied valence states by performing X-ray Absorption calculations on water clusters using Density Functional Theory. For each water cluster, studying how valence electronic structure is perturbed by changes in the local hydrogen bonding environment facilitates our description of the hydrogen bond. Also in this framework, we move toward a depiction of local structures in liquid water by comparison to experimental X-ray absorption spectra. We find consistent localization along internal bonds in the electronic structures of pre- and post-edge states for single-donor species. In addition, we propose a molecular orbital bonding-antibonding picture to explain this directional localization from dimer calculations, and show that the pre- and post-edge spectral regions have a resulting relationship.
\end{abstract}

\title{
INTRODUCTION
}

Water is central to countless biological and chemical processes in nature. Despite its extensive importance and its crucial role in living organisms, however, the structure and chemistry of liquid water are not fully understood. Liquid water molecules are associated through a hydrogen bonding network, formed as a result of internal polarization of covalent bonds. The hydrogen bond is much weaker than internal covalent bonds, but strong enough that well-defined structures could form in liquid water. 
A model that proposes organized structure in water is a sharp departure from the traditional continuum model of a disordered molecular network, but is supported by the work of [1]. Addressing the question of local structure and organization in water depends strongly upon a deeper understanding of the hydrogen bond. Its intermediacy in strength between strong covalent and weak intermolecular forces, as well as cooperativity effects, has made the electronic nature of the hydrogen bond difficult to characterize.

The application of X-ray Absorption Spectroscopy (XAS) to liquid water is a promising development toward characterization of the hydrogen bond [2]. XAS locally probes the nature of the unoccupied electronic states, which have been shown to be sensitive to changes in the hydrogen bonding network [3]. Figure 1 shows the molecular

Figure 1: Molecular Orbital Diagram for Water Monomer

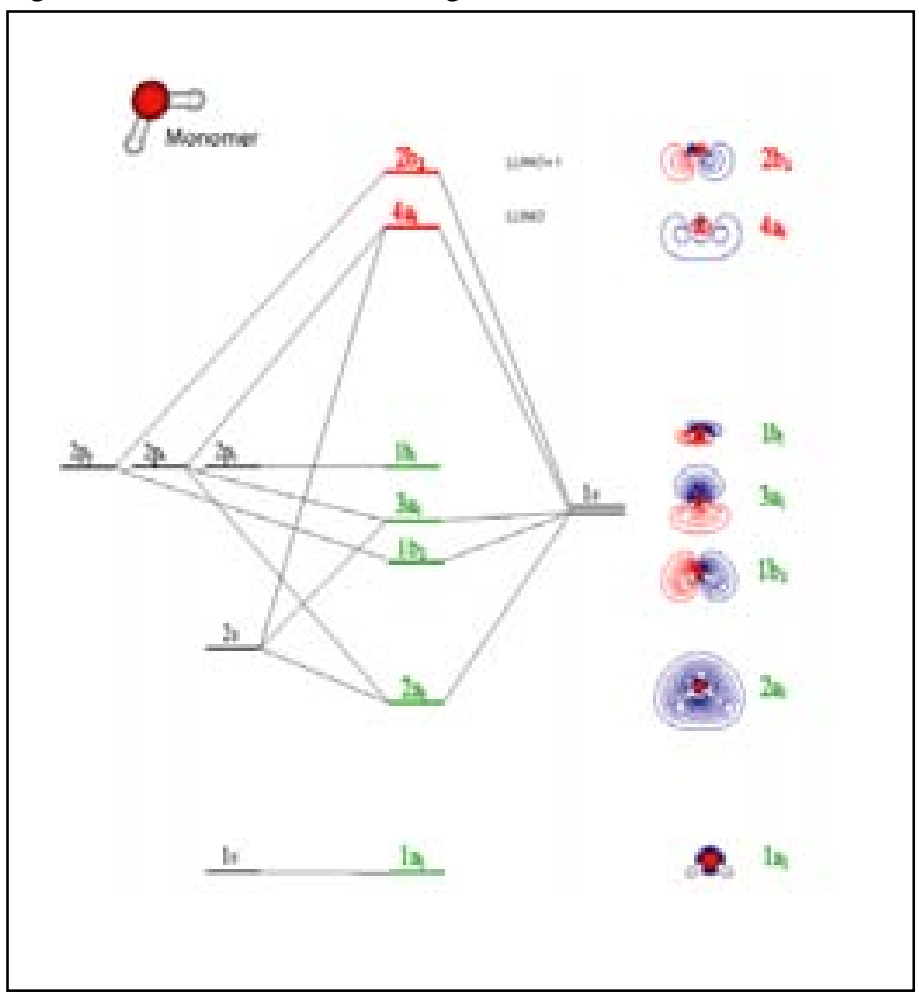

Fully occupied states (labeled in green) localize near oxygen atoms while unoccupied states (red) localize near hydrogens. The LUMO and LUMO+1 exhibit pure $a_{1}$ and $b_{2}$ symmetries, respectively. orbital diagram for a free water molecule. Due to internal polarization of the molecular $\mathrm{OH}$ bond, occupied molecular orbitals tend to localize near the highly electronegative oxygen atom, while the unoccupied orbitals localize near the hydrogen atoms [4].

For different hydrogenbonding environments, XAS can be used to locally probe the nature of unoccupied states 
through excitation of a core O1s electron. From the simple dipole selection rule, transitions are allowed into p-like states. Transition intensities reflect the extent of pcharacter in the unoccupied orbitals, and information regarding the mixing and symmetry of these orbitals can be gained. Thus, XAS is an experimentally sensitive technique to locally study the symmetry-resolved character of unoccupied states in different hydrogenbonding configurations [5]. Additionally, core electron excitations take place on the attosecond timescale, orders of magnitude faster than molecular motions in liquid water. This advantage allows investigation of electronic structure in frozen molecular geometries [6].

In this study, theoretical calculations are performed to generate X-ray absorption spectra based on density functional theory (DFT). DFT is an ab initio quantum chemistry approach which was introduced as an alternative to wavefunction based methods. Underlying DFT is the principle that a system's ground state energy, and other expectation values of observables, are functionals of the electron density $\rho(\mathbf{r})$. The variational principle for total energy holds within DFT, giving energy as a function of $\rho$ such that $E[\rho] \geq E\left[\rho_{0}\right][7]$. This method yields the ground state energy as a function of electron density through a series of self-consistent field calculations. Additionally, electron correlation and exchange energy are accounted for as functionals of electron density [5].

Dipole transition elements are obtained using the dipole operator for solved states of transition. The dipole approximation holds, where the wavelength of light is orders of magnitude larger than atomic dimensions. This allows for a simplification of transition elements to the form $\left|<\varphi_{\mathrm{f}}\right| p\left|\varphi_{\mathrm{i}}>\right|^{2}$, where $p$ is the dipole operator. This simplification 
yields atom-like selection rules, wherein the excited O1s electron is allowed transition into a p-like orbital [5]. Through the dipole selection rule, intensities of transition provide a direct method to resolve the p-character of the unoccupied valence orbitals.

The unoccupied valence states are here investigated through DFT calculations of small systems of water clusters. We begin with a water monomer and build up the hydrogen bonding network to larger clusters (up to 11 molecules) in effort to understand the changing local electronic structure that results from various bonding environments. Focus has been placed on single-donor species, in effort to concentrate on configurations we believe contribute most significantly to structure in liquid water [1]. At each step, we compare calculated spectra to the experimental spectra of liquid water. This extended approach will eventually allow exclusion of some structures as primary contributors to the overall liquid water structure, and brings others forth in contention.

\section{METHODS}

Density Functional Theory calculations were performed on small water clusters. Clusters were chosen such that the immediate hydrogen-bonding environment of the central water molecule was varied, placing emphasis

Figure 2: Ice-like Geometry on single-donor species. All clusters were arranged in a fixed ice-like geometry, where the water molecule to be excited was associated with others to reflect the tetrahedral arrangement of ice, as shown in Figure 2. All $\mathrm{HOH}$ bond angles were fixed at $109.5^{\circ}$, and all

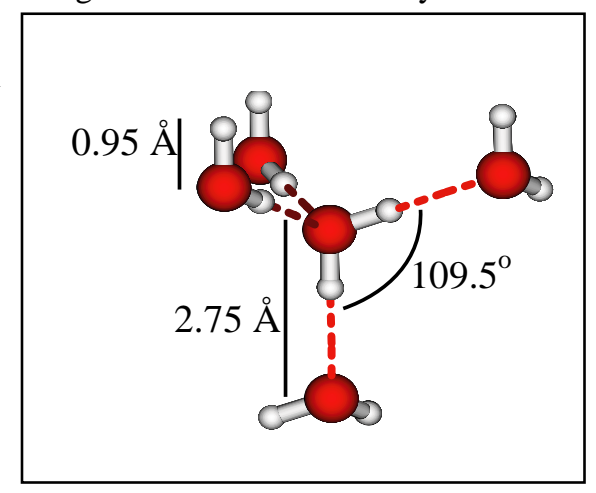


internal oxygen-hydrogen bond distances were 0.95 Á. Oxygen-oxygen (hydrogen bond) distances were fixed at $2.75 \AA$. In all cases, the coordinate system was chosen as shown

Figure 3: Coordinate system

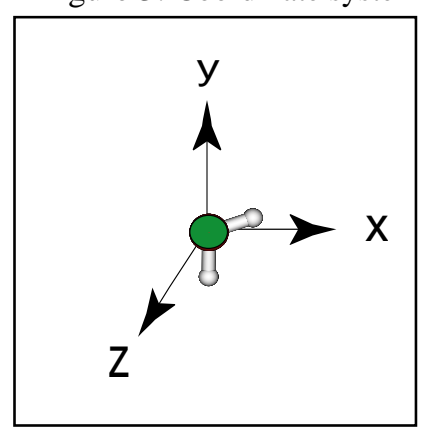

in Figure 3, where the three atoms of the excited water molecule define the xy plane and one $\mathrm{OH}$ bond is in the pure y-direction.

All computed X-ray absorption spectra were generated using the StoBe-deMon DFT code [8]. The calculations involved creation of a core hole by removing

half an electron from the 1s orbital of the central oxygen atom. This approach has been shown to generate reliable XA spectra from DFT calculations [9]. The StoBe-deMon code employs a double basis set procedure to describe electron density with Gaussian Type Orbitals of the general form $\sigma=N x^{a} y^{b} z^{c} \exp \left(-\alpha r^{2}\right)$. The first basis set describes the initial electron density prior to excitation, while a larger, more diffuse (19s, 19p, 19d) basis set describes the density in extended regions above the ionization potential for the core-excited oxygen [5]. The core-excited oxygen was described with the full-electron III_IGLO basis set [10]. Effective core potentials were used to describe the chemically inert core electrons of all other oxygen atoms.

The transition matrix elements were obtained in each direction and in total. An $\mathrm{r}^{2}$ operator was used to compute the density of s-like states and evaluate relative s contributions to solved energy states. The total oscillator strengths were broadened using gaussian functions of increasing full width at half maximum (fwhm) to simulate experimental spectra, unless otherwise noted. 


\section{RESULTS AND DISCUSSION}

For all single-donor type clusters, a visible pre-edge is observed in the generated $\mathrm{X}$-ray absorption spectra, though the relative intensities and energy positions corresponding to the LUMO and LUMO+1 vary widely and the pre-edge is seldom sharply defined. Across these configurations, we see similarities in electronic structure of states contributing to the pre-edge. Figure 4 shows a representative example of orbital pictures from a single-donor water dimer. The LUMO resembles an $\mathrm{a}_{1}$ symmetry, distorted slightly toward the free $\mathrm{OH}$ bond and delocalized from interaction with the other water molecule. The LUMO+1 orbital localizes along the free $\mathrm{OH}$ bond.

Figure 4: Pre-edge States for Donating Water Dimer

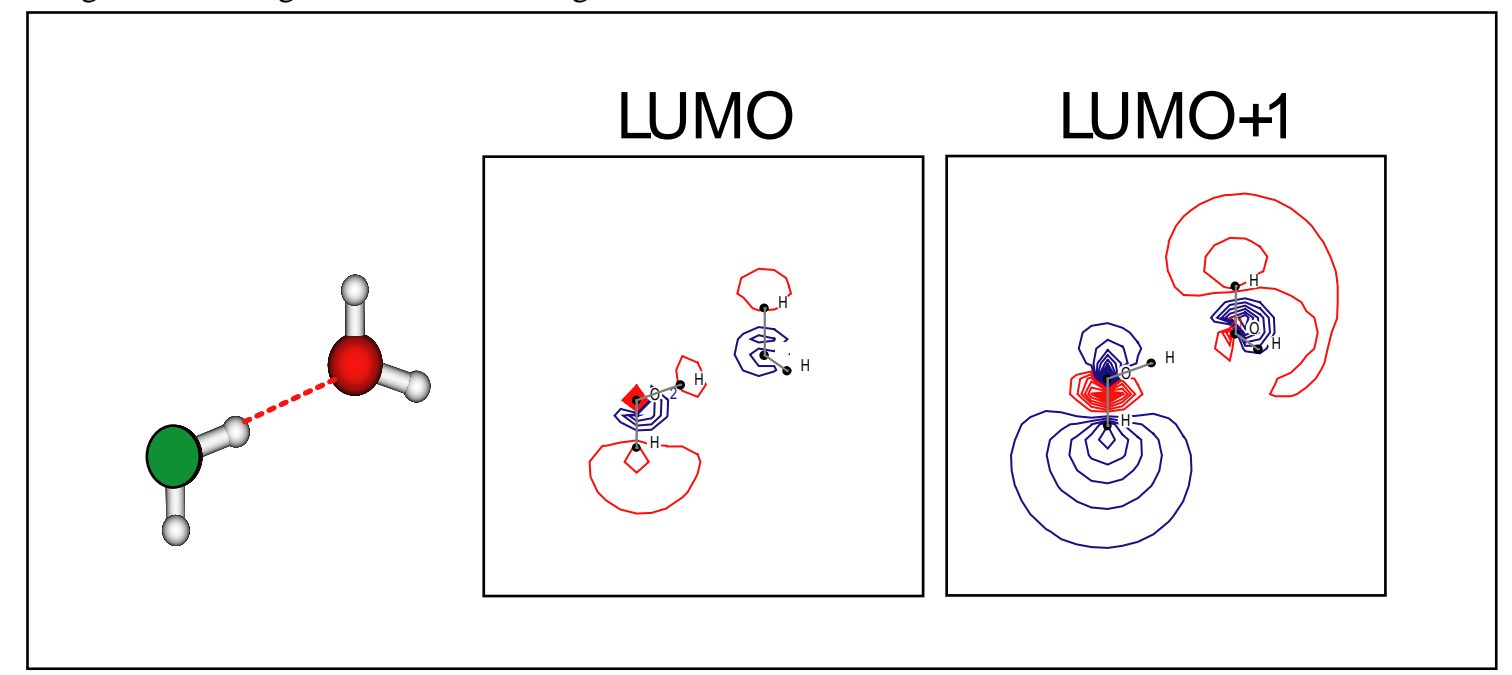

The excited water molecule (green) donates a hydrogen bond. The LUMO exhibits a slightly distorted $\mathrm{a}_{1}$ symmetry, and the LUMO+1 density is directional and more highly localized along the free $\mathrm{OH}$ bond of the donating water molecule.

Figure 5 shows an example of a computed spectra for an octamer with a welldefined pre-edge due to suppression of the LUMO oscillator strength (Figure 5; A). When water molecules are selectively removed from the second shell on the accepting side of the central water, the LUMO oscillator strength gains intensity (Figure 5; B, C). 
Simultaneously, the transition intensity for the LUMO+1 decreases. Increasing localization along the free $\mathrm{OH}$ bond is observed in the LUMO, with increasing delocalization in the LUMO+1 from the octamer to the hexamer. Calculations of larger clusters in which free $\mathrm{OH}$ bonds were closed with donating hydrogen bonds confirmed that observed delocalization was not due to surface effects. We can thus obtain a preliminary understanding of pre-edge character by observing the symmetry origins of the states to be of $a_{1}$ and $b_{2}$ symmetries. More study is needed to connect changes in the hydrogen bonding configuration with the corresponding changes in electronic structure.

Figure 5: Pre-edge spectral regions and electron density states for selected octamer, heptamer, and hexamer

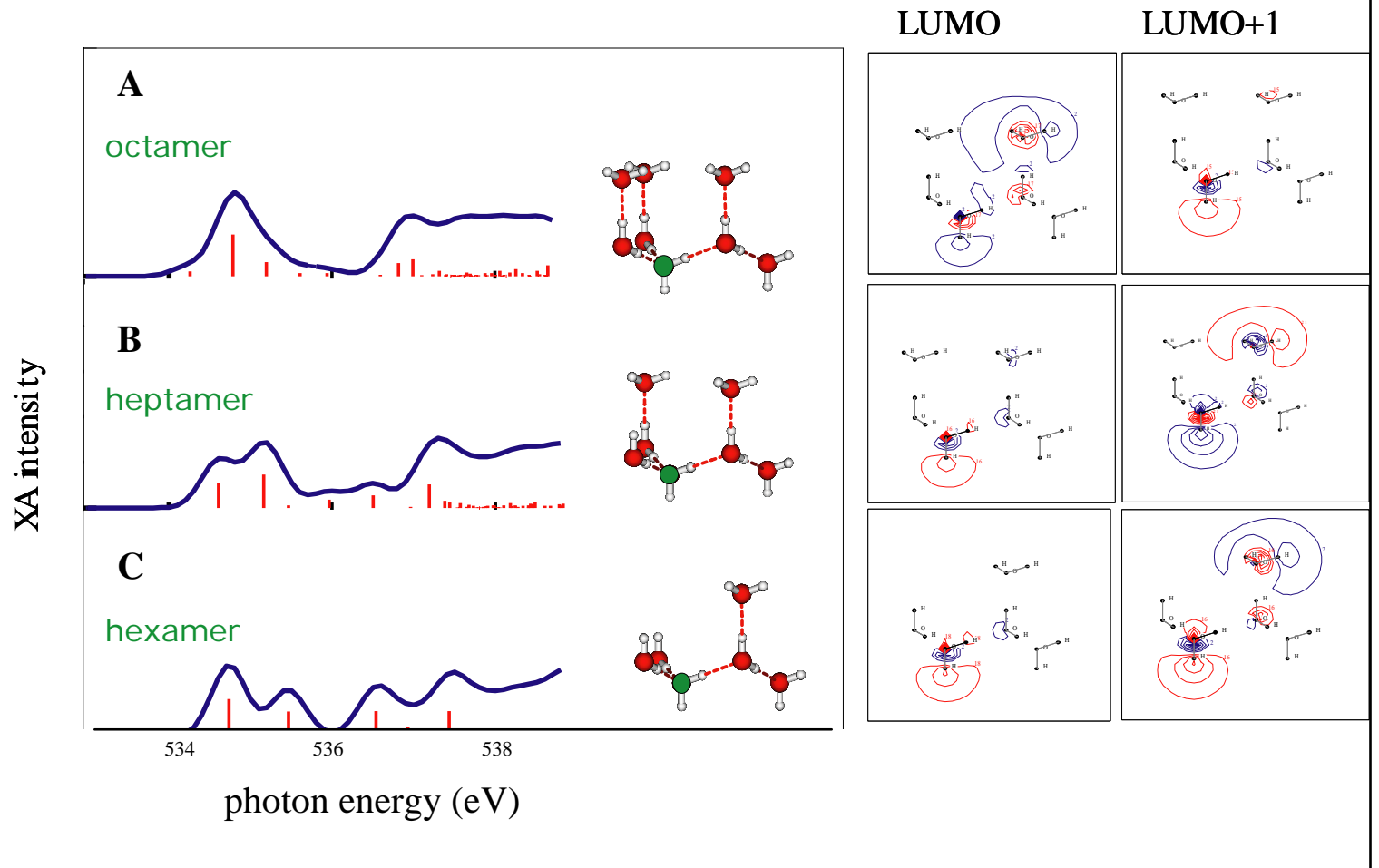

The pre-edge sharpness in the octamer spectra is lost as water molecules on the accepting side of the excited water are removed and there is a higher intensity transition to the LUMO and a weaker transition to the LUMO+1 state. 
Computed spectra for a water dimer, where the distance between oxygen atoms varies and the central molecule donates a hydrogen bond, are shown in Figure 6. In these cases, the spectra have been broadened with gaussians of a constant fwhm of $0.5 \mathrm{eV}$ to emphasize strong transitions. An increasing separation in energy is observed between the pre-edge and a sharp post-edge as the water molecules are brought closer together. The

Figure 6: Dimer Calculations for Different Hydrogen-bonding Distances

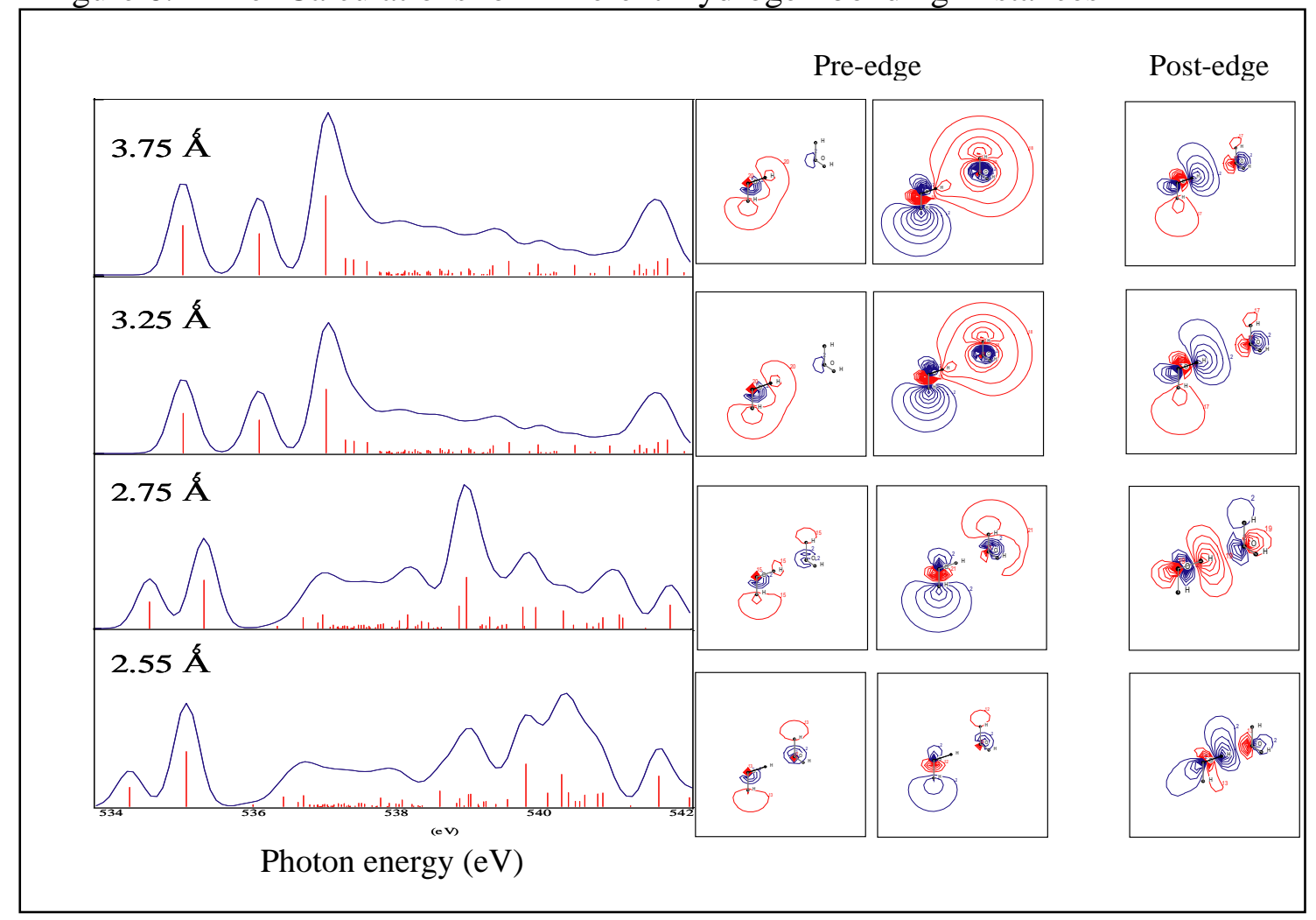

As the Oxygen-oxygen distance is decreased, greater molecular orbital overlap leads to a mixing of symmetries (observed as localization along internal $\mathrm{OH}$ bonds) and greater splitting of bonding and anti-bonding combinations (observed as increasing distance between the pre- and post-edges).

pre-edge orbitals (LUMO and LUMO+1) and the orbital corresponding to the highest oscillator strength in the post-edge region are shown alongside their spectra. Clear localization along the free $\mathrm{OH}$ bond is observed in the LUMO+1 orbitals, and localization along the donating $\mathrm{OH}$ bond is observed in the post-edge orbitals. This localization 
reflects a loss of $b_{2}$ symmetry in the LUMO+1 orbitals, and an increase in $\mathrm{py}_{\mathrm{y}}$ character. Similarly, the localized orbitals in the post edge reflect mixing of $a_{1}$ and $b_{2}$ type symmetries. We can understand these observations in electronic structure with a bonding-antibonding description of molecular orbital interaction between the two water molecules, as shown in Figure 7. As the water molecules are brought closer together, there is greater overlap of their molecular orbitals, and the interaction produces bonding-

Figure 7: Molecular Orbital Mixing in the Water Dimer

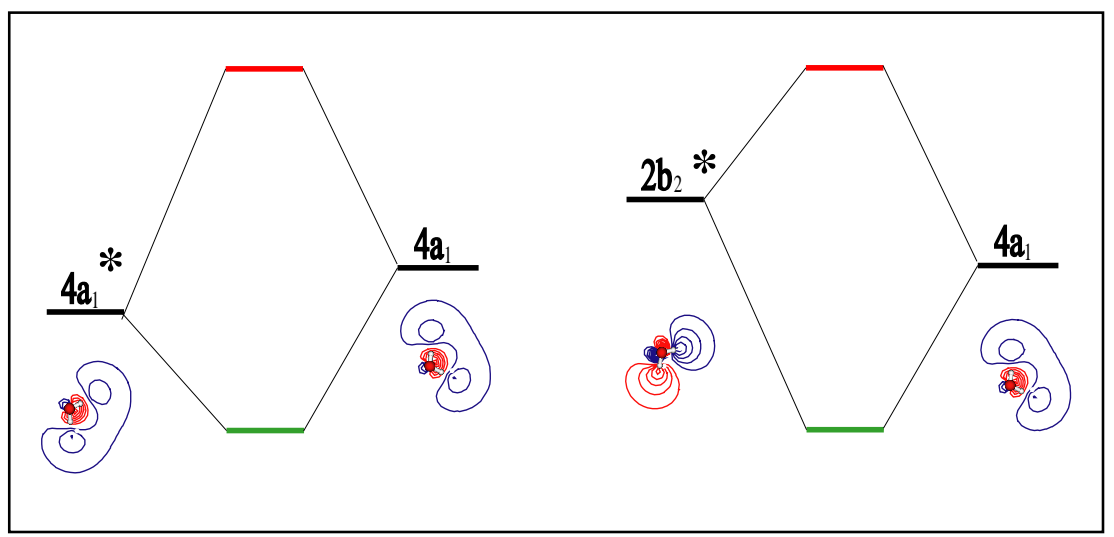

Symmetry mixing can be understood as bonding and antibonding combinations from the mixing of the $4 \mathrm{a}_{1}$ and $2 \mathrm{~b}_{2}$ excited states (denoted by asterisks) with the $4 \mathrm{a}_{1}$ state of the accepting water molecule. antibonding orbitals

which are observed as

localized states with

mixed symmetries. At

shorter distances, the

energy splitting

between bonding and

antibonding

combinations increases as molecular orbital overlap increases. We see the spectral expression of the bonding and antibonding interactions as an increasing separation between pre-edge and post-edge peaks, in Figure 6.

\section{CONCLUSION}

We have found similarities in electronic structure for unoccupied valence states of all single-donor species computed. Specifically, we see that LUMO states are of a slightly distorted $\mathrm{a}_{1}$ symmetry toward the free $\mathrm{OH}$ bond, and the LUMO+1 is localized 
directly along the free $\mathrm{OH}$ bond when there is sufficient interaction of molecular orbitals. We have begun to decipher the nature of the pre-edge in terms of the symmetry origins and localization patterns of the LUMO and LUMO+1 states. We have proposed a simple bonding-antibonding picture to describe this localization by a mixing of core-excited molecular orbitals with other valence states, observing that the localized states along the free $\mathrm{OH}$ bond contribute to pre-edge intensities, while localized states along the donating $\mathrm{OH}$ bond contribute strongly to post-edge transitions.

\section{ACKNOWLEDGMENTS}

This research was conducted at the Stanford Synchrotron Radiation Laboratory in Menlo Park, CA. I would like to thank Professor Anders Nilsson for his mentorship and guidance, and for his continued support as I continue my work on this research project. I would also like to thank Dennis Nordlund for his daily time, guidance, and teaching during the course of the summer. I extend my thanks to the Office of Science, the Department of Energy, and the Stanford Linear Accelerator Center for their support of the SULI program and for giving me the opportunity to participate.

\section{REFERENCES}

[1] Ph. Wernet, et. al., "The Structure of the First Coordination Shell in Liquid Water,” Science, Vol. 304, Issue 5673, 14 May 2004, pp. 995-999.

[2] A. Nilsson, et. al., "The hydrogen bond in ice probed by soft x-ray spectroscopy and density functional theory,” Journal of Chemical Physics, Vol. 122, April 2005, 154505.

[3] J. Stöhr, NEXAFS Spectroscopy, Berlin, Heidelberg: Springer-Verlag, 1992. 
[4] M. Cavalleri, et. al., "The interpretation of X-ray absorption spectra of water and ice,” Chemical Physics Letters, Vol. 364, 2002, pp. 363-370.

[5] D. Nordlund, "Core Level Spectroscopy of Water and Ice," Philosophy Doctor Thesis. Stockholm University; Stockholm, Sweden, 2004.

[6] S. Myneni, et. al., "Spectroscopic probing of local hydrogen-bonding structures in liquid water,” Journal of Physics: Condensed Matter, Vol. 14, 2002, pp. L213L219.

[7] W. Koch and M. C. Holthausen, A Chemist's Guide to Density Functional Theory, Weinheim: Wiley-VCH, 2002.

[8] K. Hermann, LGM Pettersson, M. Casida, C. Daul, A. Goursot, A. Koester, E. Proynov, A. St-Amant, and D. Salahub, STOBE Software, 2002.

[9] Cavalleri, et. al., "Half or full core hole in density functional theory X-ray absorption spectrum calculations of water?" Physical Chemistry Chemical Physics, Vol. 7, Issue 15, 2005, pp. 2854-2858.

[10] W. Kutzelnigg, U. Fleischer, and M. Shindler, NMR—Basic Principles and Progress, Heidelberg: Springer, 1990. 